\title{
Septin4 Aggravates Hypoxia-Induced Cardiomyocytes Injury by Promoting HIF-1a Ubiquitination and Degradation through VHL
}

\section{Shaojun Wu}

the First Hospital of China Medical University https://orcid.org/0000-0001-8662-0494

\section{Ying Zhang}

the First Hospital of China Medical University

\section{Shilong You}

the First Hospital of China Medical University

Sien Lu

the First Hospital of China Medical University

\section{Naijin Zhang}

the First Hospital of China Medical University

\section{Yingxian Sun ( $\nabla$ yxsun@cmu.edu.cn )}

the First Hospital of China Medical University

\section{Research}

Keywords: Septin4, HIF-1a, von Hippel-Lindau protein (VHL), Ubiquitination, Cardiovascular diseases

Posted Date: October 21st, 2020

DOI: https://doi.org/10.21203/rs.3.rs-95025/v1

License: () (1) This work is licensed under a Creative Commons Attribution 4.0 International License. Read Full License 


\section{Abstract}

Backgrounds: Myocardial ischemia, characterized by insufficient nutrients and oxygen supply, is the most common cause of ischemic heart disease. Hypoxia-induced cardiomyocytes injury is the pathogenic feature of myocardial ischemia. Although previous studies have reported that the proapoptotic protein Septin4 contributes to prevent some cancers by promoting tumor cells apoptosis mainly through X-linked inhibitors of apoptosis (XIAPs), little is known its role in hypoxia-induced cardiomyocytes injury and its other new interacting partner.

Results: In the current study, Septin4 is found to be involved in cardiomyocytes injury to hypoxia. The overexpression of Septin4 significantly aggravated hypoxic cardiomyocytes apoptosis assessed by cell viability assay and flow cytometry analysis. Mechanistically, hypoxia-inducible factor 1 alpha (HIF-1a) is confirmed as a novel protein mainly binding with GTPase domain of Septin4 by co-immunoprecipitation. Additionally, we found that the protective factor HIF-1 $\mathrm{a}$ is down-regulated by Septin 4 and the underlying mechanism is the von Hippel-Lindau protein (VHL)-mediated ubiquitin-proteasome degradation.

Conclusions: These findings suggest that Septin 4 aggravates hypoxia-induced cardiomyocytes injury by promoting HIF-1 a ubiquitination and degradation by targeting to VHL, which may be beneficial to provide effective strategies for clinical treatment of myocardial ischemia and ischemic heart disease.

\section{Background}

Ischemic heart disease (IHD) causes approximately 10 million deaths worldwide each year and the IHD patients' number is expected to increase by $46 \%$ by $2030[1]$. The most common cause of IHD is myocardial ischemia, a pathological state that can't supply adequate blood, which leads to the ischemic and hypoxic injury of myocardium [2-4]. Hypoxia, that is, the reduction of available oxygen, has a huge impact on the metabolism of the heart and then changes the normal heart function[5].

Hypoxia-inducible factor 1 (HIF-1) is a major transcription factor mediating cellular adaptation to hypoxia by regulating both the distribution and utilization of oxygen[6]. Actually, it is HIF-1a, the oxygen-regulated subunit of HIF-1, that activates HIF-1 under hypoxia[6]. Willam $\mathrm{C}$ et al. reported an elevated expression levels of HIF-1a protein in the rat heart after ligation of the coronary artery [7]. Kido $\mathrm{M}$ et al. found that the persistent overexpression of HIF-1 a resulted in decreased myocardial infarct size and improved murine heart function after four weeks' myocardial infarction [8]. Pre-treatment with a HIF-1a activator (dimethyloxalylglycine, OMDG) in animal modelled after ischemic-reperfusion showed an improved heart function $[9,10]$. These reveal that HIF-1 a plays a role in cardioprotection.

Septin4, a protein localized at mitochondrion, is shown to be a proapoptotic protein[11]. XIAP (X-linked IAP), a member of inhibitors of apoptosis (IAPs) family regulating cell apoptosis, inhibit apoptosis by the direct inhibition of caspases $[12,13]$. Up to date, it has been well studied that Septin4 promotes cell apoptosis mainly by binding XIAP [14-16] via the XIAP/BIR3 domain [17, 18]. Recently, BAX [19] and Bcl$2[20,21]$ are also found to be the substrate of Septin4 to promote apoptosis in some tumor cells. 
However, it is still unclear whether Septin4 participates in cardiomyocytes injury. In addition, whether there is another completely new substrate for Septin4 in cardiomyocytes also needs further research.

For the first time, our study confirmed that the expression levels of Septin4 were increased from hypoxiainduced cardiomyocytes. Knockdown of Septin4 alleviated cardiomyocytes apoptosis, but overexpression of Septin4 on the basis of Septin4 silencing aggravated it. Mechanistically, we first confirmed that HIF-1a was a novel protein binding with Septin 4 mainly via the GTPase domain of the latter. In addition, HIF-1a was down-regulated through the VHL-E3 ubiquitin ligase complex-proteasome pathway mediated by Septin4. These findings could be beneficial to provide effective strategies for clinical treatment of myocardial ischemia and IHD.

\section{Results}

\section{Septin4 participates in hypoxia-induced cardiomyocytes injury}

Given that no study has evaluated the effect of hypoxia-induced cardiomyocytes injury on Septin4 expression so far, we subjected H9c2 cells to hypoxia treatment for $0,6,12$ and 24 hours to establish hypoxia-induced injury. Successful establishment of the hypoxic model was confirmed by the observation of an obviously decreased cells viability (Additional file 1 Supplemental Fig. 1c) and a significantly increased cells apoptosis rate (Additional file 1 Supplemental Fig. 1d, e). By western blot analysis, we found obviously increased expression levels of Septin4, HIF-1a and cleaved caspase3 with the prolonging of hypoxic time (Additional file 1 Supplemental Fig. 1a, b). Thus, Septin4 may play a role in the hypoxia-induced injury model, which was discussed in the following experiments on overexpression and knockdown of Septin4 in H9c2 cells.

Cell viability assay and flow cytometry analysis showed that overexpression of Septin4 significantly aggravated hypoxia-induced H9c2 apoptosis (Fig. 1c and Fig. 1d, e), while knockdown of Septin4 by using the identified most efficacious Septin4 siRNA sequence (Fig. 1f) significantly alleviated hypoxiainduced H9c2 apoptosis (Fig. 1h and Fig. 1i, j). In addition, overexpression of Septin4 significantly increased hypoxia-induced $\mathrm{H} 9 \mathrm{c} 2$ cells apoptosis maker cleaved caspase3 protein expression (Fig. 1a, b), while knockdown of Septin4 significantly decreased cleaved caspase3 (Fig. 1f, g), and HIF-1a showed an opposite trend.

Taken as a whole, these results demonstrated that Septin4 played a negative role in cardiomyocytes survival under hypoxia.

\section{Septin4 participates in hypoxia-induced cardiomyocytes injury by down-regulating expression levels of HIF-1a}

The increase in HIF-1a expression levels by hypoxia induces HIF-1 activity, which is quite important in protecting cardiomyocytes from ischemic heart disease [22-24]. We next explored the role of Septin4 in the expression changes of HIF-1a in H9c2 cells under hypoxia. First, we found that the expression levels 
of both Septin4 and HIF-1a increased with the prolonging hypoxic time (Fig. 1a, b). Second, we evaluated the effect of Septin4 on HIF-1a in hypoxic H9c2 cells and found that the expression levels of HIF-1a significantly increased from hypoxic $\mathrm{H} 9 \mathrm{c} 2$ cells with stably silenced of Septin4, while markedly decreased after the overexpression of Septin4 in H9c2 cells on the basis of stably silenced of Septin4 (Fig. 2a, b).

At the same time, we observed an opposite expression trend of the apoptosis maker cleaved caspase3 compared to that of HIF-1a in each group (Fig. 2a, b). In addition, cell viability assay (Fig. 2c) and flow cytometry analysis (Fig. 2d, e) showed that the hypoxia-induced apoptosis was relieved with stably silenced of Septin4, while was aggravated with overexpression of Septin4 on the basis of stably silenced of Septin4.

These findings suggested that Septin4 was involved in hypoxia-induced cardiomyocytes injury by downregulating HIF-1a.

\section{Septin4 interacts with HIF-1a mainly through its GTPase domain}

To explore the mechanism of Septin4 in cardiomyocytes injury, we investigated whether Septin4 and HIF$1 \mathrm{a}$ actually interact, which then affected the injury to H9c2 cells. First, results of endogenous coimmunoprecipitation demonstrated that Septin4 is a novel protein interacting with HIF-1a (Fig. 3a, b). Next, we found that under hypoxic stimulation, the binding effect of Septin4 and HIF-1a was enhanced (Fig. 3c, d). These findings suggested that Septin4 participated in hypoxia-induced cardiomyocytes injury by interacting with HIF-1a.

Furthermore, to explore which domain of Septin4 bound with HIF-1a, four truncated plasmids of Septin4 were produced according to their functional domains (Fig. 3e). We then overexpressed full-length Septin4 or various truncated mutants of it in $293 \mathrm{~T}$ cells for co-immunoprecipitation, the results demonstrated that Septin4 mainly interacted with HIF-1a via its GTPase domain (Fig. 3f).

\section{Septin4 mediates the proteasome degradation of HIF-1a}

To further to confirm whether Septin4 affected the expression of HIF-1a, Flag-Septin4 was transfected in $\mathrm{H} 9 \mathrm{c} 2$ cells with an increasing amount. We found that the expression of Septin4 increased while the expression of HIF-1a decreased (Fig. 4a, b). What's more, the knockdown of Septin4 with the three Septin4 siRNA sequences resulted in an obviously decreased expression of Septin4 but a significantly increased expression of HIF-1a (Fig. 4c, d).

The above two findings suggested that Septin4 may mediate the degradation of HIF-1a in some way. Therefore, we investigated whether septin 4 affected the stability of HIF-1a by the use of protein synthesis inhibitor $\mathrm{CHX}$ to inhibit transcription activity or proteasome inhibitor MG132 to inhibit the proteasome degradation activity.

In the $\mathrm{CHX}$ assay, transfection of Flag-Septin4 in $\mathrm{H} 9 \mathrm{c} 2$ cells led to an obvious reduction in the expression of endogenous HIF-1 a compared to the empty vector group (Fig. 4e, f), while in the MG132 assay with 
Flag-Septin4 transfected in H9c2 cells, the accumulation of HIF-1 a was more faster and marked than the empty vector group (Fig. 4g, h), suggesting that Septin4 reduced the expression of HIF-1a by the proteasome degradation pathway.

\section{Septin4 mediates the polyubiquitination of HIF-1a}

Given that HIF-1a commonly degraded by proteasome[25], we then transfected Flag-Septin4 in H9c2 cells whether followed by the treatment of MG132 or not, and found that the interaction between Septin4 and HIF-1a was enhanced by MG132 (Fig. 5a). The results of endogenous co-immunoprecipitation assay also showed a MG132 enhanced interaction between Septin4 and HIF-1a (Fig. 5b).

Furthermore, after co-transfecting Flag-Septin4 and HA-UB in H9c2 cells followed by the treatment of MG132, we found that Septin4 significantly increased HIF-1a ubiquitination levels by coimmunoprecipitation assay (Fig. 5c). What's more, Septin4 silencing was found to reduce HIF-1a ubiquitination levels in Septin4 knockdown H9c2 cells after overexpression of HA-UB (Fig. 5d). These results suggested that Septin4-mediated degradation of HIF-1a by the proteasome pathway was dependent on polyubiquitination.

\section{Septin4 promotes the ubiquitin-proteasome degradation of HIF-1a by mobilizing von Hippel-Lindau protein ( $\mathrm{VHL})$}

The existence of VHL in an E3 ubiquitin ligase complex, which can mediate the rapid proteasome degradation of HIF-1 a, makes HIF-1 a levels low on normoxia [26, 27]. We next bought HIF-PHDs inhibitor BAY85-3934, which negatively affect the VHL-HIF-1a interaction and then clarified whether Septin4 mediates the ubiquitin-proteasome degradation of HIF-1a via VHL.

First, we evaluated the effect of Septin4 and BAY85-3934 on HIF-1a in hypoxic H9c2 cells and found that the expression levels of HIF-1a significantly decreased under hypoxia after the overexpression of Septin4, while BAY85-3934 can rescue this result (Fig. 6a, b). Second, to explain the phenomenon, we then transfected Flag-Septin4 in H9c2 cells and found that the interaction between HIF-1a and VHL was enhanced (Fig. 6c) while Septin4 silencing was found to reduce this binding effect. (Fig. 6d). Lastly, after co-transfecting Flag-Septin4 and HA-UB in H9c2 cells, we found that BAY85-3934 significantly decreased the ubiquitination levels of HIF-1a (Fig. 6e).

These results confirmed that Septin4 mediated HIF-1a polyubiquitination via VHL and promoted HIF-1a degradation by the proteasome pathway (Fig. 6f).

\section{Discussion}

The main findings of the current study are that, Septin4 is first found to interact with HIF-1a mainly via its GTPase domain and negatively regulates HIF-1a through the ubiquitin-proteasome pathway by enhancing the binding effect between von Hippel-Lindau protein (VHL) and HIF-1a. In the hypoxic 
cardiomyocytes, Septin4 had obviously increased expression levels and enhanced binding effect with HIF-1a, which result in significant reduction of HIF-1a and then aggravated cardiomyocytes apoptosis.

Sept4_i2 (ARTS) is one of the splice variants encoded by Septin 4 gene, and is the only one with the proapoptotic function [28]. In recent years, several studies have shown that Septin4 may be a target of preventing cardiovascular diseases. Zhang et al. [29] first reported that Setpin4 aggravated oxidative stress induced endothelial cell apoptosis by interacting with PARP1. Another study[30] performed by Zhang et al. showed that Septin4 protected human vascular smooth muscle cells from phenotypic transformation, proliferation and migration induced by PDGF-BB by promoting SIRT1-mediated STAT3 acetylation at $\mathrm{K} 685$ site and phosphorylation at $\mathrm{Y} 705$ site. These two findings provided a theoretical basis of the treatment of atherosclerosis. In addition, Wang et al. [31] found that Septin4 inhibited Ang-II induced vascular smooth muscle cells proliferation and migration. Our previous study[32] revealed that WWP2 bound with Septin4 in human umbilical vein endothelial cells and degraded it through the ubiquitin-proteasome pathway at K174 site of Septin4 to prevent hypertensive angiopathy. The present study first confirmed that the expression levels of Septin4 were increased from hypoxia. Knockdown of Septin4 decreased cardiomyocytes apoptosis while overexpression of Septin4 on the basis of Septin4 silencing increased it. To explore the phenomenon, we found HIF-1a, which is beneficial to adaptations to hypoxia, binding with the GTPase domain of Septin4 and was down-regulated by Septin4.

HIF-1a and HIF-1 $\beta$ are the two subunits of HIF-1, in which HIF-1a subunit plays the central role in protecting cells from local hypoxia by increasing expression of its downstream target genes in many cardiovascular diseases [5] . The target genes of HIF-1a are those involved in angiogenesis, vascular reactivity (including secreted factors and cell surface receptors) and glucose metabolism (including metabolic enzymes, transporters and mitochondrial proteins) [6, 33]. For example, neovascularization stimulated by expression of vascular endothelial growth factor (VEGF) is induced by HIF-1a mediated transcription of VEGF gene on hypoxia [34]. In addition, injection of AdCA5, an adenovirus providing an active form of HIF-1 a showed the improved vascular remodeling and angiogenic responses [35-37]. In the present study, we performed rescue experiment, CHX, MG132 and poly-ubiquitination assays and found the stability of the protective factor HIF-1 a was negatively affected by Septin4 through the ubiquitinproteasome pathway, which suggested that Septin4 indeed aggravated the hypoxia-induced cardiomyocytes injury by down-regulating HIF-1a.

Under normoxia, HIF-1a is continually synthesized and rapidly degraded by the hydroxylation at Pro402 or Pro564 within the oxygen-dependent degradation domain (ODDD) particularly by PHD2 [38] before VHL, the recognition subunit of an E3 ubiquitin ligase complex, targets the hydroxylated HIF-1a for proteasome degradation [39]. And under hypoxia, the accumulation of HIF-1a results from the inhibited proline residues hydroxylation and the following ubiquitination and proteasome degradation [39]. In the current study, we found BAY85-3934 (a PHDs inhibitor) significantly inhibited the down-regulation and ubiquitination levels of HIF-1 a mediated by Septin4. Additionally, overexpression of Septin4 showed enhanced interaction between HIF-1 $a$ and VHL while Septin4 silencing reduced the binding effect. This confirmed the molecular mechanism of down-regulation of HIF-1a by Septin4 was targeted to VHL- 
mediated proteasome degradation, which explained how Septin4 was involved in hypoxia-induced cardiomyocytes injury in detail.

There are some limitations of the present study. First, although HIF-1a does have a protective effect through its target genes under hypoxic condition, we have not yet determined which gene played the protective role in our experiment. Second, the role of Septin4 in myocardial ischemic patients remains to be further studied.

\section{Conclusions}

Septin4 is found to be involved in hypoxia-induced cardiomyocytes injury and HIF-1a is confirmed as a novel protein mainly binding with the GTPase domain of Septin4. Additionally, HIF-1a is down-regulated by Septin 4 and the underlying mechanism is the VHL-mediated ubiquitin-proteasome degradation. These findings may be beneficial to provide effective strategies for clinical treatment of myocardial ischemia and ischemic heart disease.

\section{Materials And Methods}

\section{Cell culture and hypoxia treatment}

The rat cardiomyocyte H9c2 cell line and the human embryonic kidney 293T cell line were procured from the Chinese Academy of Sciences (Shanghai, China) and cultured in DMEM supplemented with $10 \%$ FBS (HyClone). All cells were situated in an incubator containing $5 \% \mathrm{CO}_{2}$ and $95 \%$ air at $37{ }^{\circ} \mathrm{C}$. To establish the hypoxia model, $\mathrm{H} 9 \mathrm{c} 2$ cells were cultured with serum-free DMEM in an incubator containing $94 \% \mathrm{~N}_{2}$, $5 \% \mathrm{CO}_{2}$, and $1 \% \mathrm{O}_{2}$ for 24 hours unless otherwise noted.

\section{Plasmids construction and transfection}

Flag-Septin4 (full length) and HA-UB were purchased from GeneChem (China). Four truncated Septin4 plasmids containing different domains with a Flag-tag were obtained: N-terminal and GTPase domains; $\mathrm{N}$-terminal domain; C-terminal domain; C-terminal and GTPase domains. Lipofectamine 3000 (Invitrogen, California, USA) was used for plasmid transfections following the manufacturer's instructions.

\section{Septin4 knockdown in $\mathrm{H} 9 \mathrm{c} 2$ cells}

Normal Control and Septin4 shRNAs were obtained from GeneChem (China). Septin4 silencing was performed with lentivirus. To prevent off-target effects, three sequences were employed:

Septin4 shRNA-1: GCGGAAGAGAGGATCATGCAA

Septin4 shRNA-2: CTGCATCAGCGGGTCAACATT

Septin4 shRNA-3: TGGCCTGAATCGCAAGAACAT 
Control and Septin4 siRNAs were obtained from RIBOBIO (China). Septin4 silencing was performed with jetPRIME transfection reagent from PolyPlus (France). To prevent off-target effects, three sequences were employed:

Septin4 siRNA-1: CGGTGGATATAGAAGAGAA

Septin4 siRNA-2: CTATCAGTTCCCAGATTGT

Septin4 siRNA-3: ATGCAAACCGTGGAGATTA

The efficiency of Septin4 knockdown by shRNA and siRNA was confirmed by Western blot analysis.

\section{Antibodies and reagents}

Polyclonal goat anti-Septin4 (abcam, USA), polyclonal rabbit anti-HIF-1a (abcam), polyclonal rabbit antiVHL (proteintech, USA), polyclonal rabbit anti-caspase-3 (Cell Signaling Technology, USA), monoclonal mouse anti-HA (Cell Signaling Technology), monoclonal mouse anti-Flag (abcam), and monoclonal mouse anti-GAPDH (abcam). CHX (A8244) and MG132 (A2585), used in DMSO, were purchased from ApexBio (USA). HIF-PHDs inhibitor BAY85-3934 was also purchased from ApexBio.

\section{Cell viability assay}

Cell viability was assessed with a Cell Counting Kit-8 assay (Dojindo, Kumamoto, Japan). Briefly, H9c2 cells were seeded in 96-well plates (NEST Biotechnology) at a density of 5000 cells/well in DMEM with $10 \%$ FBS. After being performed the indicated treatments, cells were incubated with $110 \mu \mathrm{l}$ of CCK-8 solution (serum-free DMEM/CCK-8 reagent $=10 / 1$ ) per well for 2 hours. Finally, the absorbance value of each well was measured at a wavelength of $450 \mathrm{~nm}$ with a Microplate ReaderBio-Rad microplate reader (Model 680; Bio Rad Laboratories, Inc., Hercules, CA, USA).

\section{Flow cytometry analysis}

To detect cell apoptosis, Annexin V-FITC/PI Kit (Beyotime Institute of Biotechnology) were used to stain treated cells according to the manufacturer's instruction, followed by flow cytometry analysis. Briefly, treated $\mathrm{H} 9 \mathrm{c} 2$ cells were collected carefully and resuspended in $500 \mu \mathrm{l}$ binding buffer containing $3.5 \mu \mathrm{l}$ Annexin V-FITC and $3.5 \mu \mathrm{l} \mathrm{PI}$ to react with $\mathrm{H} 9 \mathrm{c} 2$ cells in the dark for $15 \mathrm{~min}$ at RT. Finally, apoptotic cells were identified and quantified with a FACSCalibur flow cytometer.

\section{Protein preparation}

For protein analysis, the H9c2 cells were washed with cold PBS, harvested and lysed as quickly as possible on ice in cell lysis buffer ( $50 \mathrm{mM}$ Tris, $137 \mathrm{mM} \mathrm{NaCl}, 1 \mathrm{mM}$ EDTA, $10 \mathrm{mM} \mathrm{NaF}, 0.1 \mathrm{mM} \mathrm{Na}_{3} \mathrm{VO}_{4}$, 1\% NP-40, 1 mM DTT, 10\% glycerol, pH 7.8) with both protease (Roche, Switzerland) and phosphatase inhibitors (Bimake, USA), then centrifuged at $13300 \mathrm{rpm} / \mathrm{min}$ for 15 minutes at $4{ }^{\circ} \mathrm{C}$. 


\section{Co-immunoprecipitation and immunoblotting}

The lysates were incubated with specific antibodies (antibody $/$ cell lysate $=1 \mu \mathrm{g} / \mathrm{mg}$ ) for 3 hours followed by adding 30-35 $\mu$ I Protein A/G beads (Bimake) and incubating for another 12 hours at $4^{\circ} \mathrm{C}$. Next, the lysates including antibodies and beads were washed with cell lysis buffer for three times and resolved by $8 \%$ or $12 \%$ SDS-PAGE gels and then transferred to the PVDF membranes (Millipore USA). Afterwards, the PVDF membranes were blocked by $5 \%$ BSA in TBST for 1 hour at RT before incubations with primary antibodies overnight at $4{ }^{\circ} \mathrm{C}$ and with secondary antibodies for 1 hour at RT the next day. Image $\mathrm{J} 1.52 \mathrm{v}$ (National Institutes of Health, USA) was used to quantify the immunoreactive bands. The expression levels of target protein were showed as fold changes relative to expression levels of corresponding GAPDH.

\section{Statistical analysis}

Data are presented as mean \pm standard deviation (SD). F-test was performed to evaluate the homogeneity of variance and the normality of data was evaluated by Shapiro-Wilk test. To determine the significance of differences between several groups of one related study factor or two, one-way ANOVA and two-way ANOVA were performed respectively, followed by Tukey's multiple comparisons test. All data were processed by SPSS 25.0 (SPSSInc, Chicago, USA) and GraphPad Prism 8 for Windows, $p<0.05$ was considered significant.

\section{List Of Abbreviations}

IHD: Ischemic heart disease

PARP1: Poly(ADP-ribose) polymerase-1

HIF-1: Hypoxia-inducible factor 1

VHL: von Hippel-Lindau protein

IAPs: Inhibitors of apoptosis XIAP (X-linked IAP)

CHX: Cycloheximide

VEGF: vascular endothelial growth factor

PHD: Prolyl hydroxylase domain proteins

ODDD: Oxygen-dependent degradation domain

\section{Declarations}

Ethics approval and consent to participate: Not applicable. 
Consent for publication: Not applicable.

Availability of data and materials: All data generated or analyzed during this study are included in this published article and its supplementary information files.

Competing interests: The authors declare that they have no competing interests.

Funding: This study was supported by grants from the "National Natural Science Foundation of China" (Grant No. 81970254, 81800381, 81670231, 81970211, and 81900327).

Authors' contribution: Yingxian Sun guided this study. Shaojun Wu conducted the cells and mechanism part of the experiments. Shilong You and Sien Lu contributed in the cells and mechanism part of the experiments. Ying Zhang and Naijin Zhang designed the experiments. Shaojun Wu and Naijin Zhang wrote the manuscript. All authors read and approved the final manuscript.

Acknowledgements: Not applicable.

\section{References}

1. Benjamin EJ, Virani SS, Callaway CW, Chamberlain AM, Chang AR, Cheng S, Chiuve SE, Cushman M, Delling FN, Deo R et al: Heart Disease and Stroke Statistics-2018 Update: A Report From the American Heart Association. Circulation 2018, 137(12):e67-e492.

2. Giordano FJ: Oxygen, oxidative stress, hypoxia, and heart failure. J Clin Invest 2005, 115(3):500-508.

3. Hsieh DJ, Huang CY, Pai P, Wang SG, Tsai YL, Li CN, Kuo WW, Huang CY: Prolactin protects cardiomyocytes against intermittent hypoxia-induced cell damage by the modulation of signaling pathways related to cardiac hypertrophy and proliferation. Int J Cardiol 2015, 181:255-266.

4. Zhang P, Ke J, Li Y, Huang L, Chen Z, Huang X, Zhang L, Xiao D: Long-term exposure to high altitude hypoxia during pregnancy increases fetal heart susceptibility to ischemia/reperfusion injury and cardiac dysfunction. Int J Cardiol 2019, 274:7-15.

5. Sousa Fialho MDL, Abd Jamil AH, Stannard GA, Heather LC: Hypoxia-inducible factor 1 signalling, metabolism and its therapeutic potential in cardiovascular disease. Biochim Biophys Acta Mol Basis Dis 2019, 1865(4):831-843.

6. Semenza GL: Hypoxia-inducible factor 1 and cardiovascular disease. Annu Rev Physiol 2014, 76:3956.

7. Willam C, Maxwell PH, Nichols L, Lygate C, Tian YM, Bernhardt W, Wiesener M, Ratcliffe PJ, Eckardt $\mathrm{KU}$, Pugh CW: HIF prolyl hydroxylases in the rat; organ distribution and changes in expression following hypoxia and coronary artery ligation. $J$ Mol Cell Cardiol 2006, 41(1):68-77.

8. Kido M, Du L, Sullivan CC, Li X, Deutsch R, Jamieson SW, Thistlethwaite PA: Hypoxia-inducible factor 1-alpha reduces infarction and attenuates progression of cardiac dysfunction after myocardial infarction in the mouse. J Am Coll Cardiol 2005, 46(11):2116-2124. 
9. Ockaili R, Natarajan R, Salloum F, Fisher BJ, Jones D, Fowler AA, 3rd, Kukreja RC: HIF-1 activation attenuates postischemic myocardial injury: role for heme oxygenase-1 in modulating microvascular chemokine generation. Am J Physiol Heart Circ Physiol 2005, 289(2):H542-548.

10. Poynter JA, Manukyan MC, Wang Y, Brewster BD, Herrmann JL, Weil BR, Abarbanell AM, Meldrum DR: Systemic pretreatment with dimethyloxalylglycine increases myocardial HIF-1a and VEGF production and improves functional recovery after acute ischemia/reperfusion. Surgery 2011, 150(2):278-283.

11. Abbas R, Larisch S: Targeting XIAP for Promoting Cancer Cell Death-The Story of ARTS and SMAC. Cells 2020, 9(3).

12. Salvesen GS, Duckett CS: IAP proteins: blocking the road to death's door. Nat Rev Mol Cell Biol 2002, 3(6):401-410.

13. Schimmer AD: Inhibitor of apoptosis proteins: translating basic knowledge into clinical practice. Cancer Res 2004, 64(20):7183-7190.

14. Garrison JB, Correa RG, Gerlic M, Yip KW, Krieg A, Tamble CM, Shi R, Welsh K, Duggineni S, Huang Z et al: ARTS and Siah collaborate in a pathway for XIAP degradation. Mol Cell 2011, 41(1):107-116.

15. Fuchs $Y$, Brown S, Gorenc T, Rodriguez J, Fuchs E, Steller H: Sept4/ARTS regulates stem cell apoptosis and skin regeneration. Science 2013, 341(6143):286-289.

16. Edison N, Zuri D, Maniv I, Bornstein B, Lev T, Gottfried Y, Kemeny S, Garcia-Fernandez M, Kagan J, Larisch S: The IAP-antagonist ARTS initiates caspase activation upstream of cytochrome $\mathrm{C}$ and SMAC/Diablo. Cell Death Differ 2012, 19(2):356-368.

17. Gottfried Y, Rotem A, Lotan R, Steller H, Larisch S: The mitochondrial ARTS protein promotes apoptosis through targeting XIAP. Embo j 2004, 23(7):1627-1635.

18. Bornstein B, Gottfried Y, Edison N, Shekhtman A, Lev T, Glaser F, Larisch S: ARTS binds to a distinct domain in XIAP-BIR3 and promotes apoptosis by a mechanism that is different from other IAPantagonists. Apoptosis 2011, 16(9):869-881.

19. Zhao X, Feng H, Wang Y, Wu Y, Guo Q, Feng Y, Ma M, Guo W, Song X, Zhang Y et al: Septin4 promotes cell death in human colon cancer cells by interacting with BAX. Int J Biol Sci 2020, 16(11):1917-1928.

20. Mamriev D, Abbas R, Klingler FM, Kagan J, Kfir N, Donald A, Weidenfeld K, Sheppard DW, Barkan D, Larisch S: A small-molecule ARTS mimetic promotes apoptosis through degradation of both XIAP and Bcl-2. Cell Death Dis 2020, 11(6):483.

21. Edison N, Curtz Y, Paland N, Mamriev D, Chorubczyk N, Haviv-Reingewertz T, Kfir N, Morgenstern D, Kupervaser M, Kagan J et al: Degradation of Bcl-2 by XIAP and ARTS Promotes Apoptosis. Cell Rep 2017, 21(2):442-454.

22. Martin C, Yu AY, Jiang BH, Davis L, Kimberly D, Hohimer AR, Semenza GL: Cardiac hypertrophy in chronically anemic fetal sheep: Increased vascularization is associated with increased myocardial expression of vascular endothelial growth factor and hypoxia-inducible factor 1. Am J Obstet Gynecol 1998, 178(3):527-534.

23. Lee SH, Wolf PL, Escudero R, Deutsch R, Jamieson SW, Thistlethwaite PA: Early expression of angiogenesis factors in acute myocardial ischemia and infarction. N Engl J Med 2000, 342(9):626- 
633.

24. Jürgensen JS, Rosenberger C, Wiesener MS, Warnecke C, Hörstrup JH, Gräfe M, Philipp S, Griethe W, Maxwell PH, Frei U et al: Persistent induction of HIF-1alpha and -2alpha in cardiomyocytes and stromal cells of ischemic myocardium. Faseb j 2004, 18(12):1415-1417.

25. Semenza GL: Hypoxia-inducible factor 1: regulator of mitochondrial metabolism and mediator of ischemic preconditioning. Biochim Biophys Acta 2011, 1813(7):1263-1268.

26. Salceda S, Caro J: Hypoxia-inducible factor 1alpha (HIF-1alpha) protein is rapidly degraded by the ubiquitin-proteasome system under normoxic conditions. Its stabilization by hypoxia depends on redox-induced changes. J Biol Chem 1997, 272(36):22642-22647.

27. Maxwell PH, Wiesener MS, Chang GW, Clifford SC, Vaux EC, Cockman ME, Wykoff CC, Pugh CW, Maher ER, Ratcliffe PJ: The tumour suppressor protein VHL targets hypoxia-inducible factors for oxygen-dependent proteolysis. Nature 1999, 399(6733):271-275.

28. Mandel-Gutfreund Y, Kosti I, Larisch S: ARTS, the unusual septin: structural and functional aspects. Biol Chem 2011, 392(8-9):783-790.

29. Zhang NJ, Zhang Y, Zhao SC, Sun YX: Septin4 as a novel binding partner of PARP1 contributes to oxidative stress induced human umbilical vein endothelial cells injure. Biochem Bioph Res Co 2018, 496(2):621-627.

30. Zhang NJ, Zhang Y, You SL, Tian YC, Lu SE, Cao L, Sun YX: Septin4 Prevents PDGF-BB-induced HAVSMC Phenotypic Transformation, Proliferation and Migration by Promoting SIRT1-STAT3 Deacetylation and Dephosphorylation. International Journal of Biological Sciences 2020, 16(4):708718.

31. Wang N, Xu F, Lu SE, Zhang NJ, Sun YX: Septin4 as an autophagy modulator regulates Angiotensin-II mediated VSMCs proliferation and migration. Biochem Bioph Res Co 2020, 525(2):272-279.

32. Zhang NJ, Zhang Y, Wu BQ, You SL, Sun YX: Role of WW domain E3 ubiquitin protein ligase 2 in modulating ubiquitination and Degradation of Septin4 in oxidative stress endothelial injury. Redox Biology 2020, 30.

33. Semenza GL: Hypoxia-inducible factor 1: oxygen homeostasis and disease pathophysiology. Trends Mol Med 2001, 7(8):345-350.

34. Forsythe JA, Jiang BH, lyer NV, Agani F, Leung SW, Koos RD, Semenza GL: Activation of vascular endothelial growth factor gene transcription by hypoxia-inducible factor 1. Mol Cell Biol 1996, 16(9):4604-4613.

35. Rey S, Lee K, Wang CJ, Gupta K, Chen S, McMillan A, Bhise N, Levchenko A, Semenza GL: Synergistic effect of HIF-1alpha gene therapy and HIF-1-activated bone marrow-derived angiogenic cells in a mouse model of limb ischemia. Proc Natl Acad Sci U S A 2009, 106(48):20399-20404.

36. Bosch-Marce M, Okuyama H, Wesley JB, Sarkar K, Kimura H, Liu YV, Zhang H, Strazza M, Rey S, Savino $L$ et al: Effects of aging and hypoxia-inducible factor-1 activity on angiogenic cell mobilization and recovery of perfusion after limb ischemia. Circ Res 2007, 101(12):1310-1318. 
37. Patel TH, Kimura H, Weiss CR, Semenza GL, Hofmann LV: Constitutively active HIF-1alpha improves perfusion and arterial remodeling in an endovascular model of limb ischemia. Cardiovasc Res 2005, 68(1):144-154.

38. Berra E, Benizri E, Ginouvès A, Volmat V, Roux D, Pouysségur J: HIF prolyl-hydroxylase 2 is the key oxygen sensor setting low steady-state levels of HIF-1alpha in normoxia. Embo j 2003, 22(16):40824090.

39. Semenza GL: Involvement of oxygen-sensing pathways in physiologic and pathologic erythropoiesis. Blood 2009, 114(10):2015-2019.

\section{Figures}



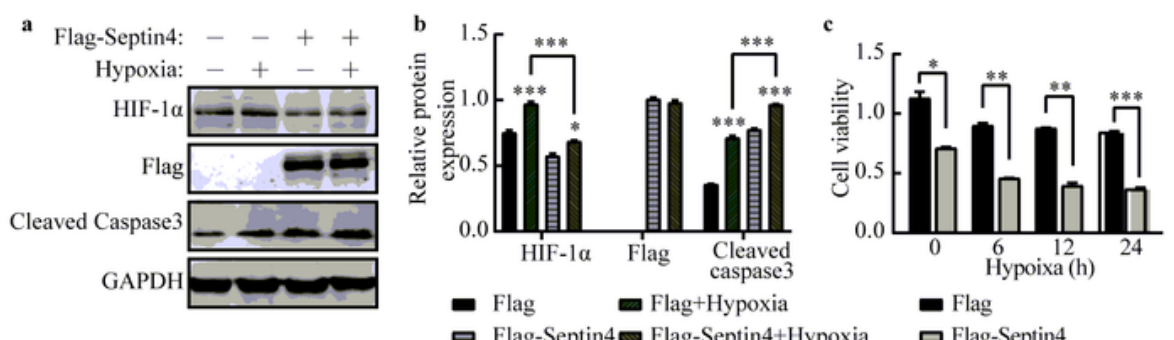

d

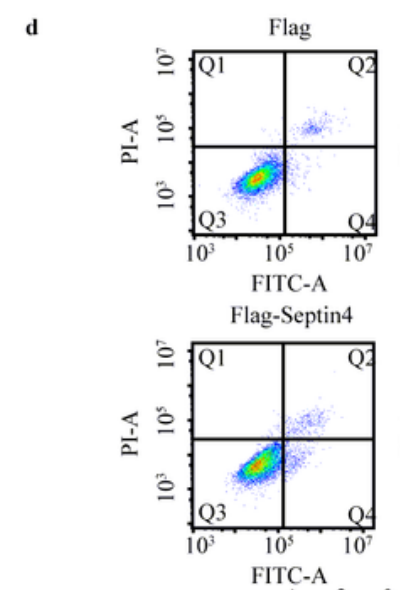

- Flag-Septin4 $\mathbf{m}$ Flag-Septin4+Hypoxia

口 Flag-Septin4

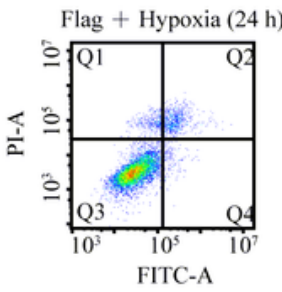

Flag-Septin4 + Hypoxia (24 h)
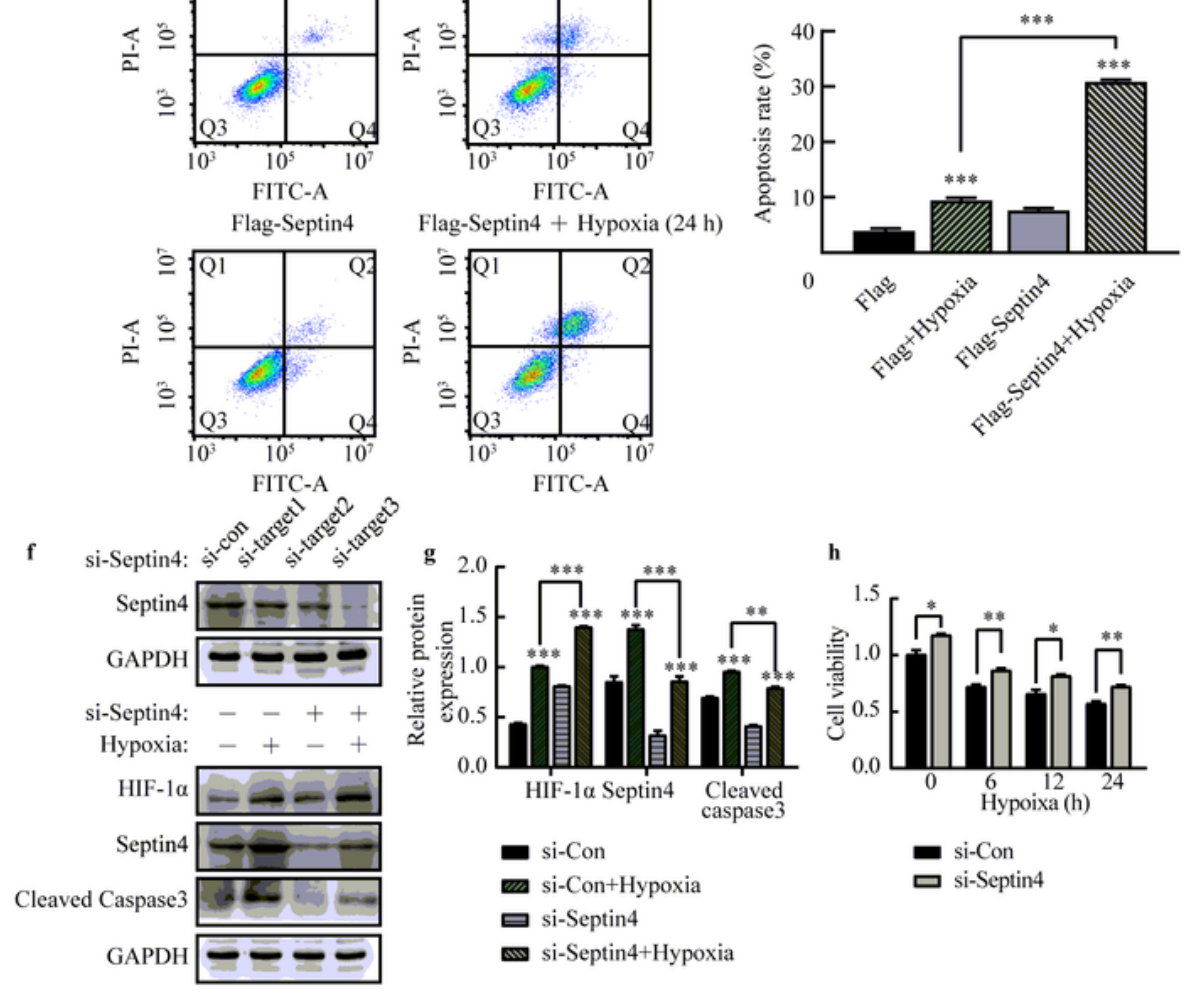

h
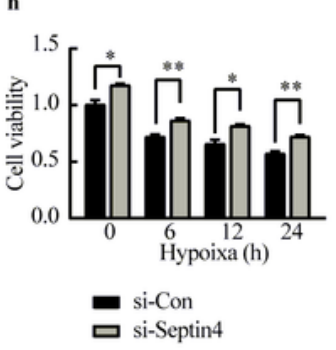

si-Septin4+Hypoxia
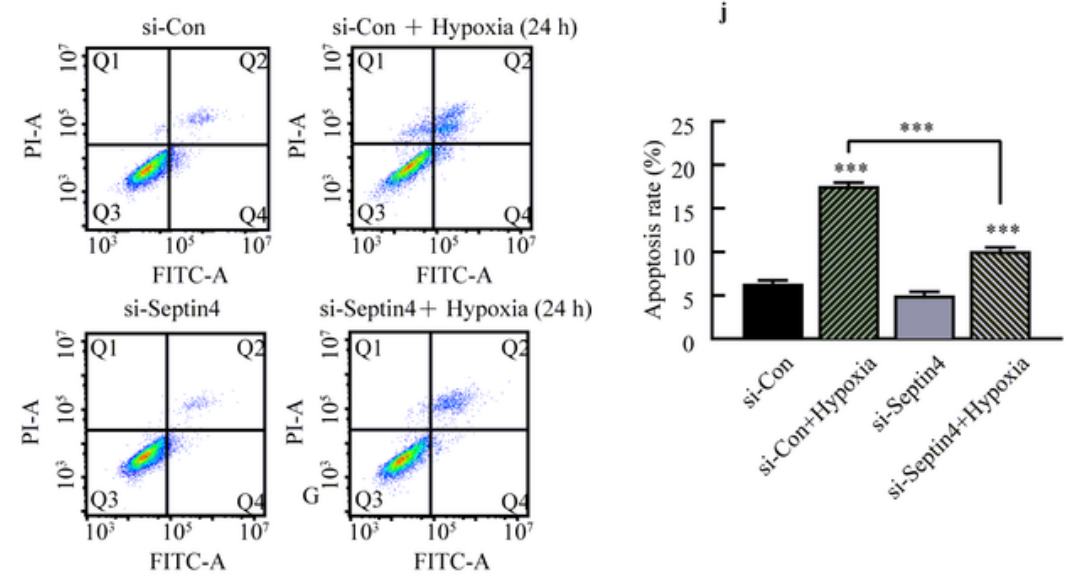

Figure 1

Septin4 participates in hypoxia-induced cardiomyocytes injury a b Western blot analysis of the expression levels of HIF-1a, Septin4 and cleaved caspases 3 in H9c2 cells transfected with vector empty or Flag-Septin4 under hypoxia for $24 \mathrm{~h}$. c Cell viability assay of $\mathrm{H} 9 \mathrm{c} 2$ cells' viability after transfected with vector empty or Flag-Septin4 under hypoxia for $24 \mathrm{~h}$. d e Flow cytometry analysis of H9c2 cells' apoptosis rate after transfected with vector empty or Flag-Septin 4 under hypoxia for $24 \mathrm{~h}$. $\mathrm{f} \mathrm{g} \mathrm{Western} \mathrm{blot} \mathrm{analysis}$ 
of the expression levels of HIF-1a, Septin4 and cleaved caspases3 in H9c2 cells transfected with si-Con or si-Septin4 under hypoxia for $24 \mathrm{~h}$. h Cell viability assay of H9c2 cells' viability after transfected with siCon or si-Septin4 under hypoxia for $24 \mathrm{~h}$. i j Flow cytometry analysis of H9c2 cells' apoptosis rate after transfected with si-Con or si-Septin4 under hypoxia for $24 \mathrm{~h}$. All data are presented as mean $\pm \mathrm{SD}$ of three repeated experiments; $* * *<0.001, * *<0.01, *<0.05$. One-way ANOVA with Tukey's multiple comparisons test (b, e, g, and j); two-way ANOVA with Tukey's multiple comparisons test (c, h).
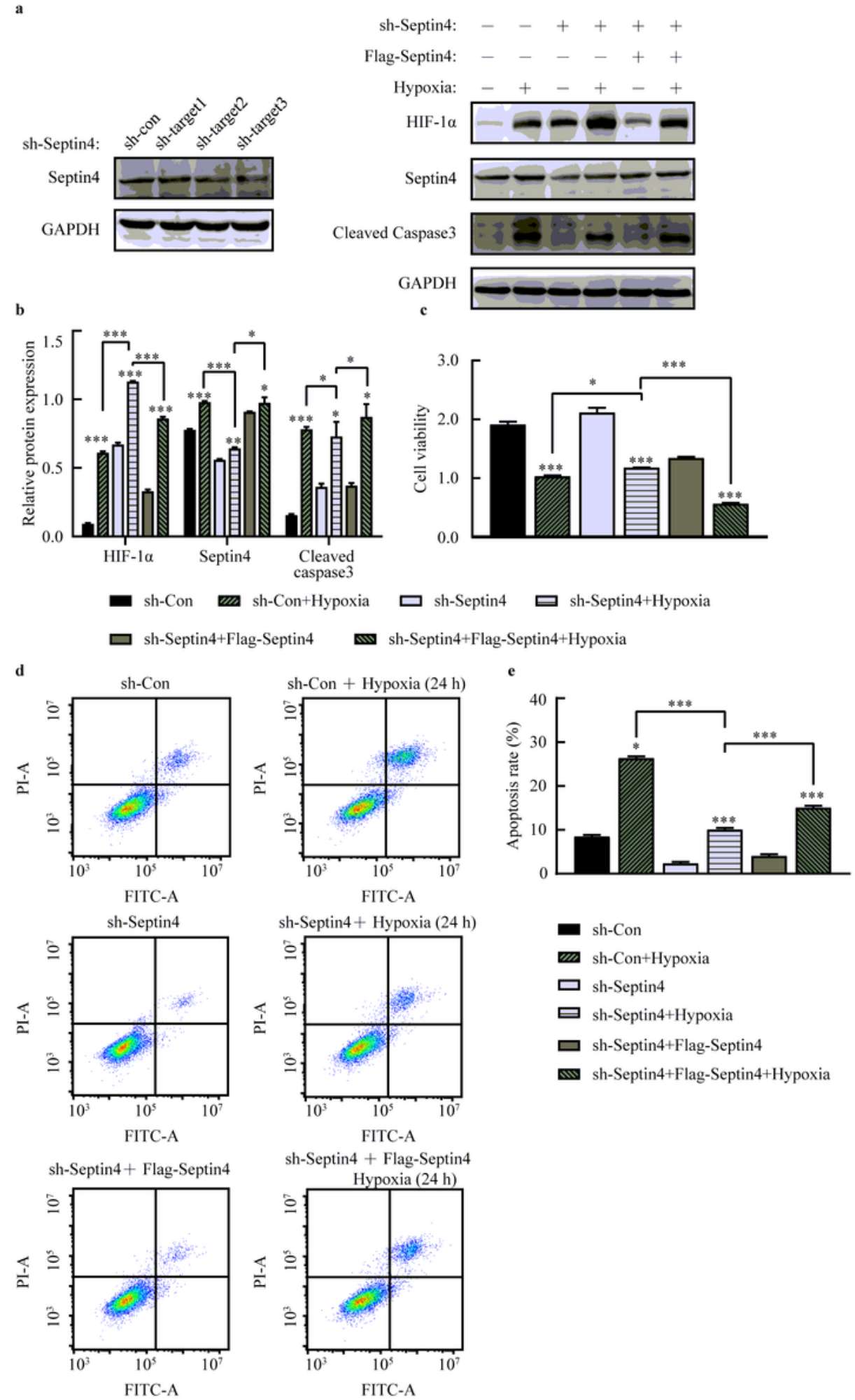

Figure 2 
Septin4 participates in hypoxia-induced cardiomyocytes injury by down-regulating expression levels of HIF-1a a b Western blot analysis of the role of Septin4 in regulating the expression levels of HIF-1a in shSeptin $4 \mathrm{H} 9 \mathrm{c} 2$ cells transfected with vector empty or Flag-Septin4 under hypoxia for $24 \mathrm{~h}$. c Cell viability assay of sh-Septin4 $\mathrm{H} 9 \mathrm{c} 2$ cells' viability after transfected with vector empty or Flag-Septin4 under hypoxia for $24 \mathrm{~h}$. d e Flow cytometry analysis of sh-Septin $4 \mathrm{H} 9 \mathrm{c} 2$ cells' apoptosis rate after transfected with vector empty or Flag-Septin 4 under hypoxia for $24 \mathrm{~h}$. All data are presented as mean \pm SD of three repeated experiments; $* \star \star<0.001, * \star<0.01, *<0.05$. One-way ANOVA with Tukey's multiple comparisons test (b, c, and e). 
a

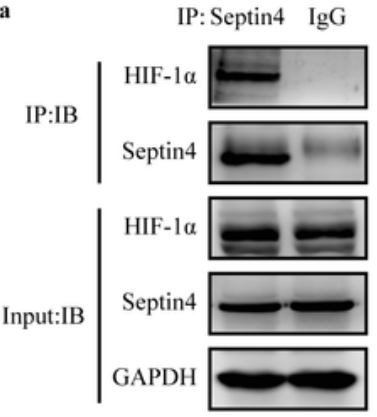

IP: Septin4 Septin4 IgG
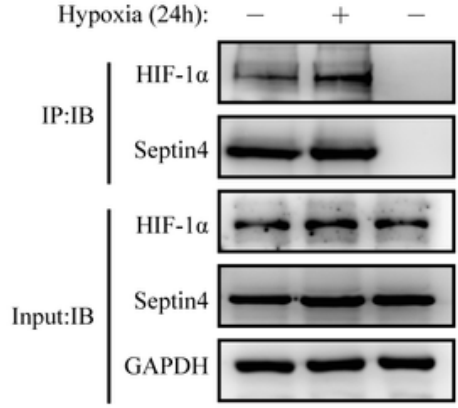

e
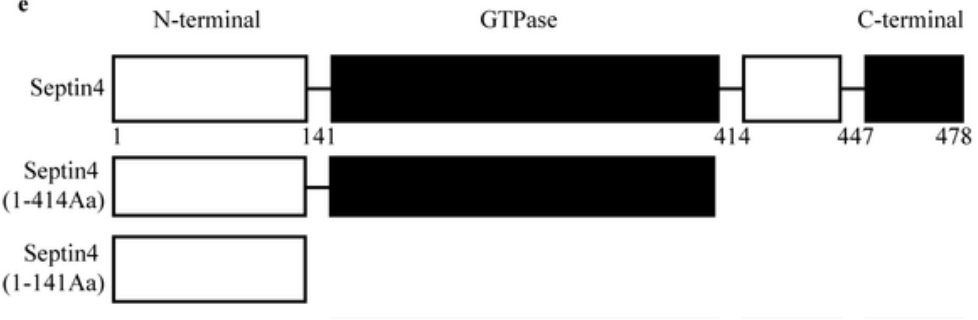

Septin4
$(414-478 \mathrm{Aa})$
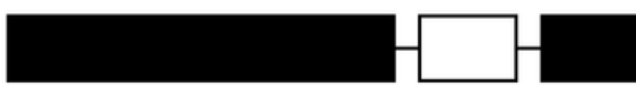

Septin4

(141-478Aa)

f

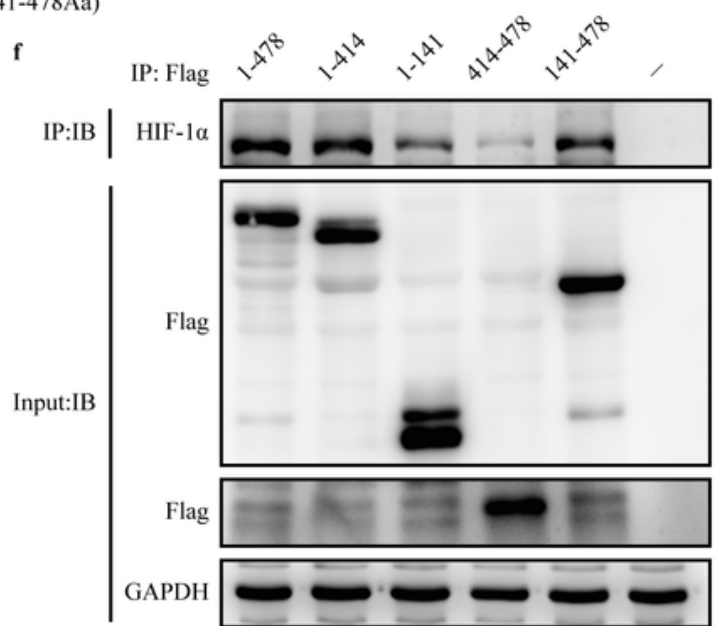

\section{Figure 3}

Septin4 interacts with HIF-1a mainly through its GTPase domain a b Co-immunoprecipitation analysis of the endogenous interaction between Septin4 and HIF-1a. c d Co-immunoprecipitation analysis of the endogenous interaction between Septin4 and HIF-1a under hypoxia for $24 \mathrm{~h}$. e f Co-immunoprecipitation analysis of the interaction between different truncations of Septin4 (exogenously overexpressed) and HIF-1a. 
a

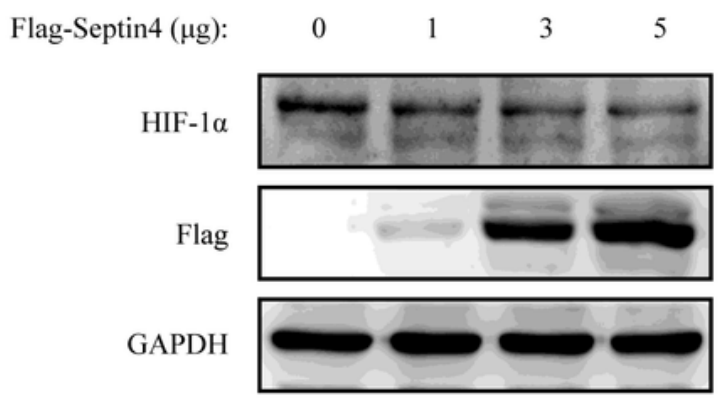

c

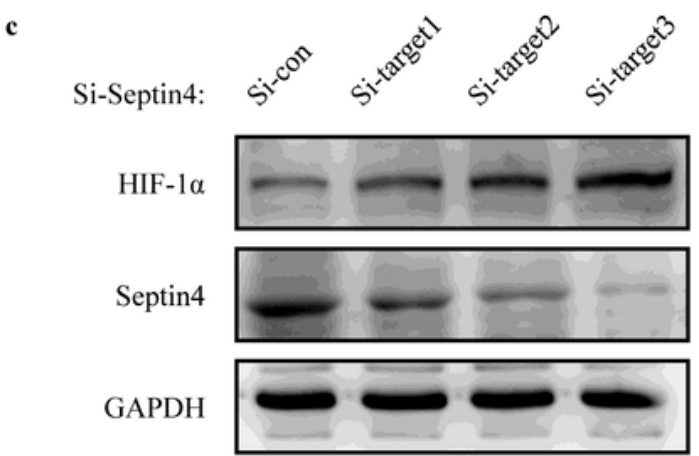

e Flag-Septin4:

$\begin{array}{lllllll}\text { CHX (h): } & 0 & 4 & 8 & 0 & 4 & 8\end{array}$

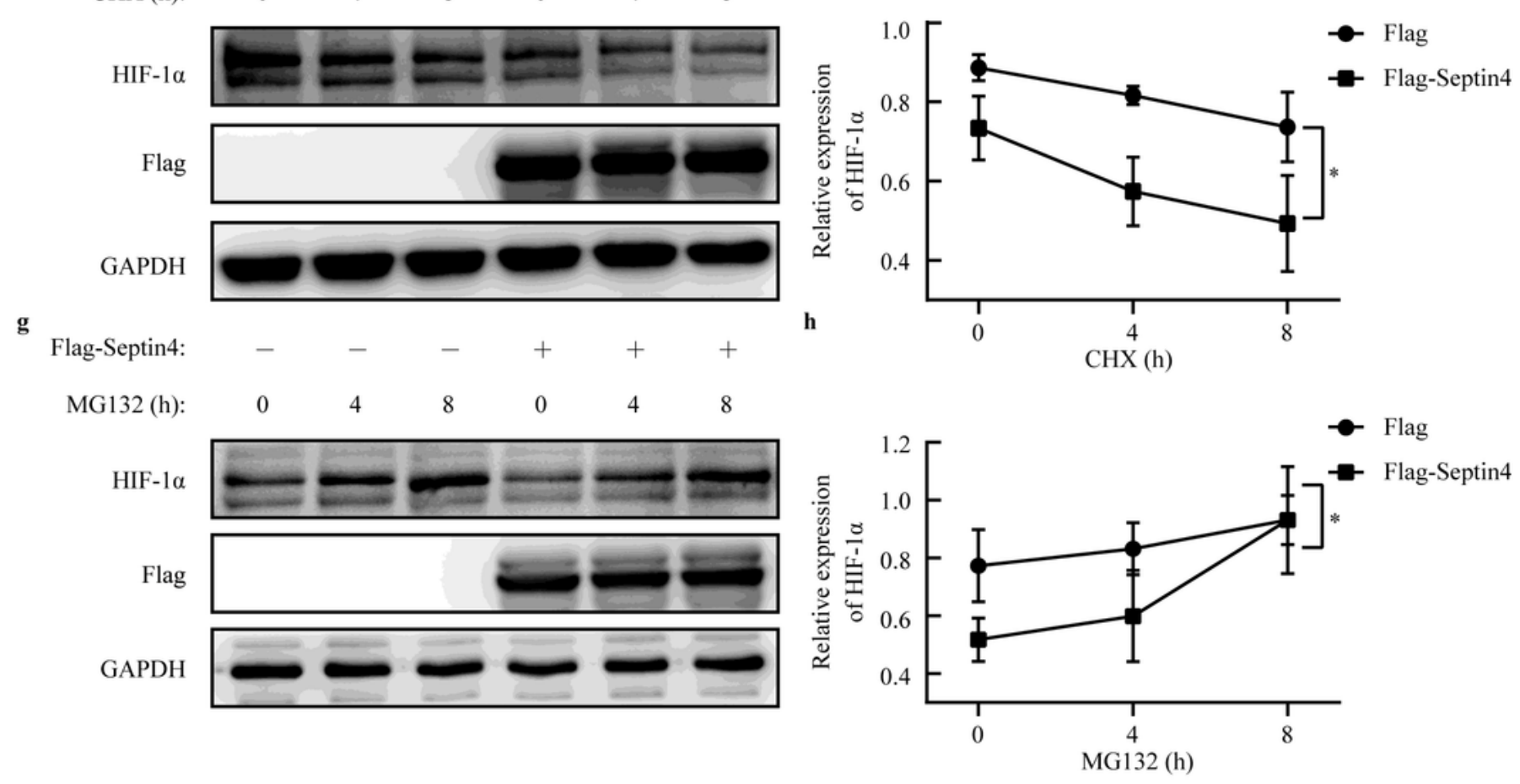

b

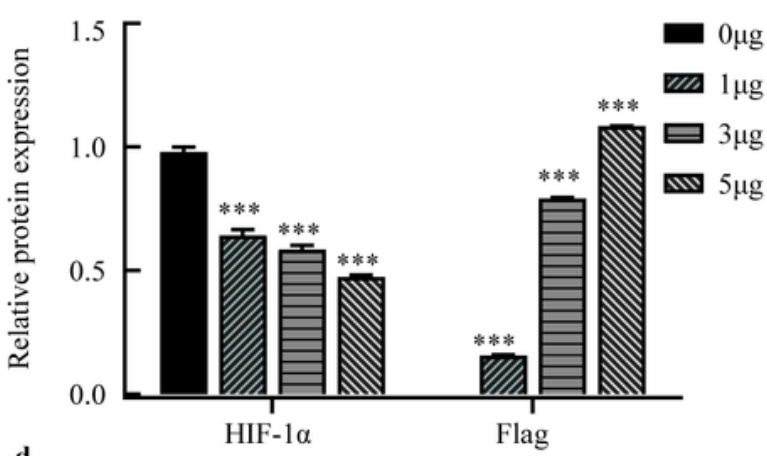

d

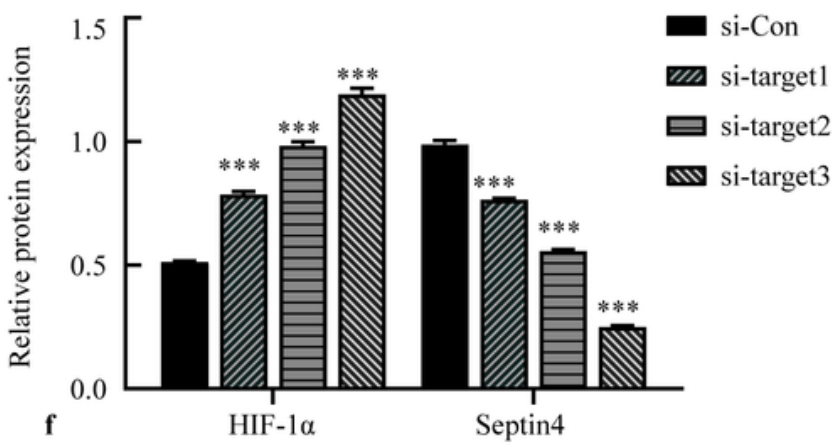

\section{Figure 4}

Septin4 mediates the proteasome degradation of HIF-1a a b Western blot analysis of the gradient overexpression of Septin4 and the changes of HIF-1a protein expression levels. $c d$ Western blot analysis of the knockdown of Septin4 with three different sequences and the changes of HIF-1 a protein expression levels e f Western blot analysis of the expression levels of HIF-1a under the treatment of CHX for different hours in H9c2 cells transfected with vector empty or Flag-Septin4. $\mathrm{g}$ h Western blot analysis of the 
expression levels of HIF-1a under the treatment of MG132 for different hours in H9c2 cells transfected with vector empty or Flag-Septin4. All data are presented as mean \pm SD of three repeated experiments; *** $<0.001, * \star<0.01, *<0.05$. One-way ANOVA with Tukey's multiple comparisons test (b, d). Two-way ANOVA with Tukey's multiple comparisons test $(f, h)$.

a
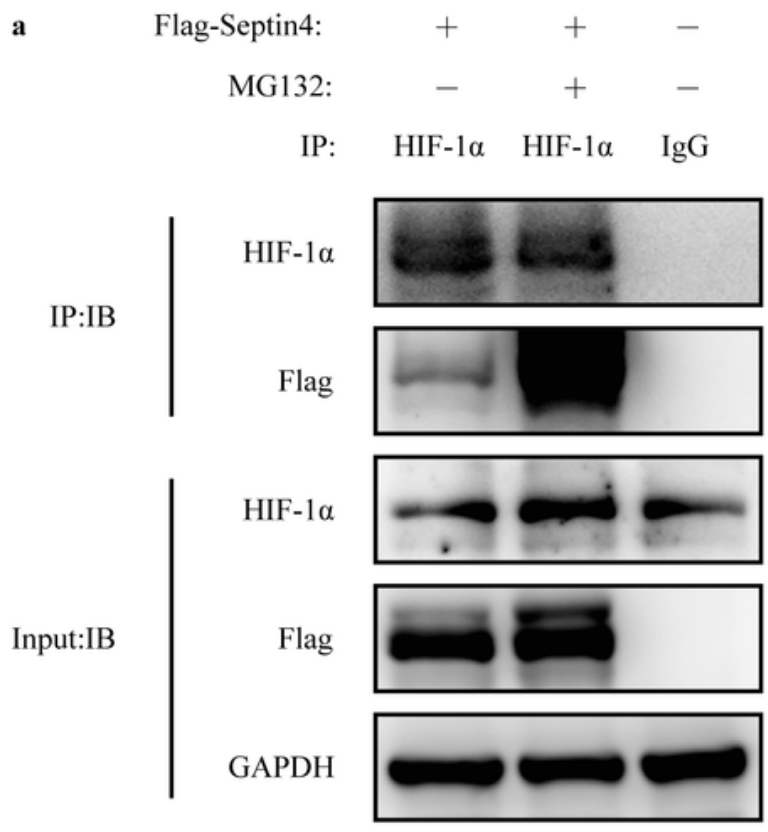

c

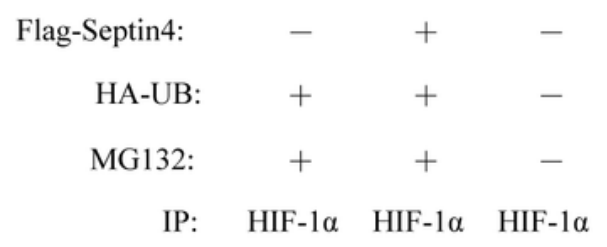

IP:IB

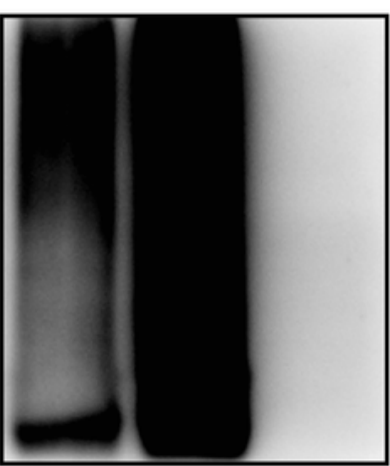

Input:IB

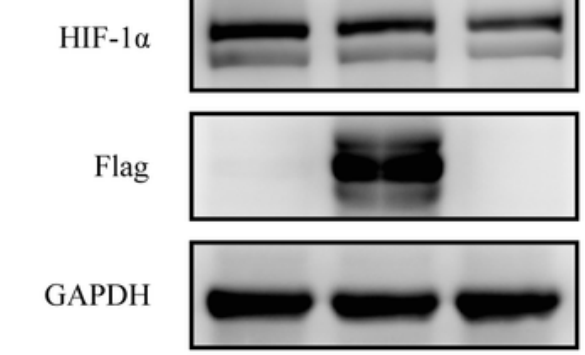

b

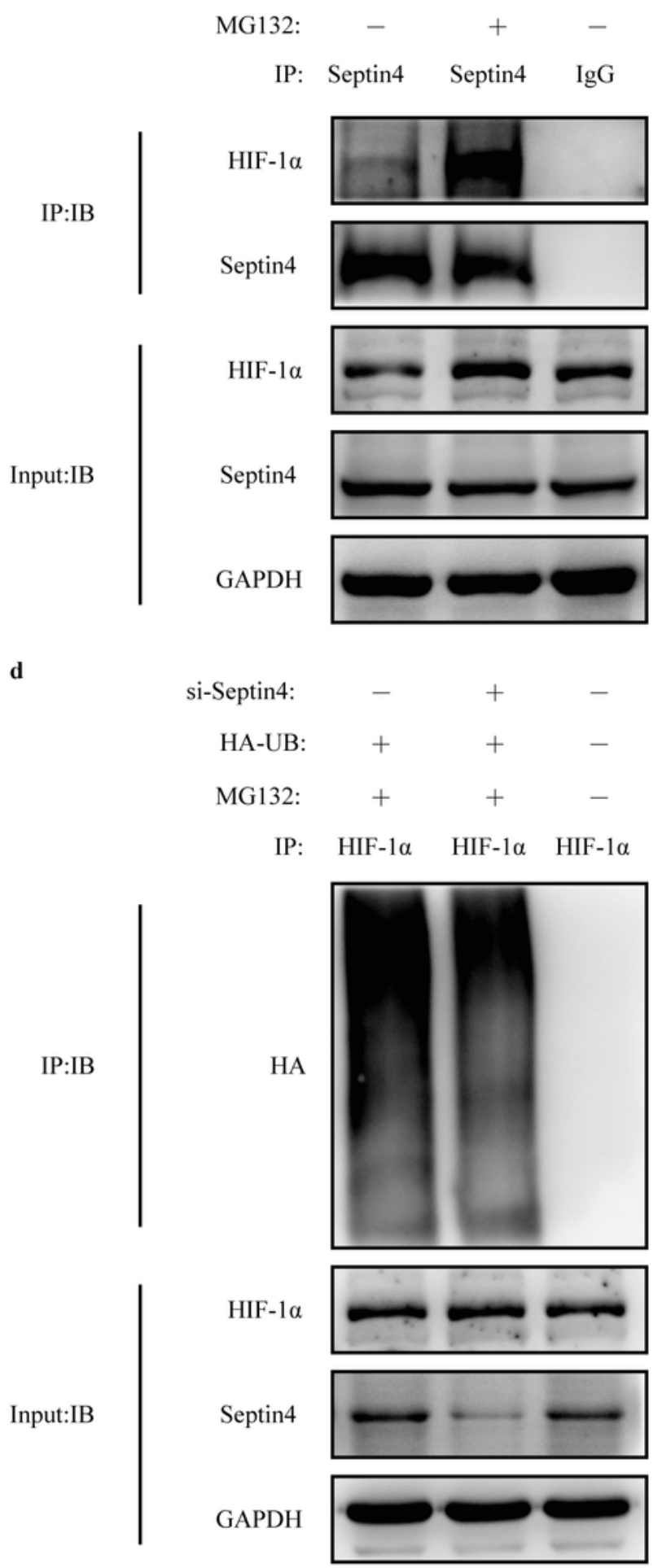

Figure 5 
Septin4 mediates the polyubiquitination of HIF-1a a Co-immunoprecipitation analysis of the interaction between exogenous Septin4 and endogenous HIF-1a under the treatment of MG132 for 8 hours. b Coimmunoprecipitation analysis of the interaction between endogenous Septin4 and endogenous HIF-1a under the treatment of MG132 for 8 hours. c Co-immunoprecipitation analysis of the role of Septin4 in regulating the ubiquitination levels of HIF-1a in $\mathrm{H} 9 \mathrm{c} 2$ cells transfected with vector empty or Flag-Septin4. d Co-immunoprecipitation analysis of the role of Septin4 in regulating the ubiquitination levels of HIF-1a in $\mathrm{H} 9 \mathrm{c} 2$ cells transfected with si-Con or si-Septin4.

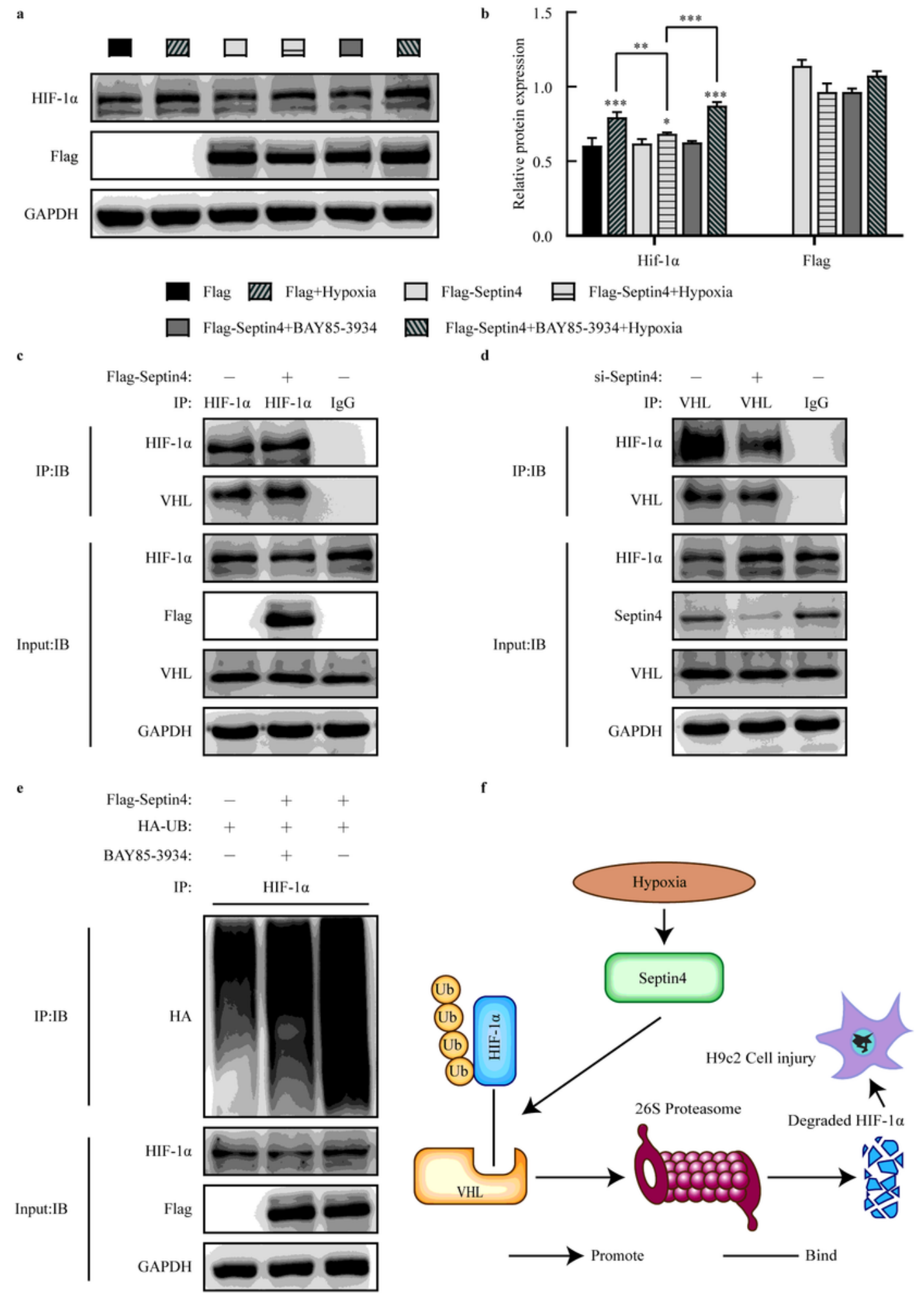




\section{Figure 6}

Septin4 promotes the ubiquitin-proteasome degradation of HIF-1a by mobilizing von Hippel-Lindau protein (VHL) a b Western blot analysis of the expression levels of HIF-1a rescued by BAY85-3934 in H9c2 cells transfected with vector empty or Flag-Septin4. c Co-immunoprecipitation analysis of the role of Septin4 in regulating the endogenous interaction between VHL and HIF-1a in H9c2 cells transfected with vector empty or Flag-Septin4. d Co-immunoprecipitation analysis of the role of Septin4 in regulating the endogenous interaction between VHL and HIF-1 a in H9c2 cells transfected with si-Con or si-Septin4. e Coimmunoprecipitation analysis of BAY85-3934's effect on the necessity of Septin4 regulating the ubiquitination levels of HIF-1a in H9c2 cells transfected with vector empty or Flag-Septin4. $f$ Proposed working mode of Septin4 as a negative regulator of HIF-1 a by targeting VHL-mediated HIF-1a ubiquitinproteasome degradation. All data are presented as mean \pm SD of three repeated experiments; $* \star \star<0.001$, ** $<0.01, *<0.05$. One-way ANOVA with Tukey's multiple comparisons test (b).

\section{Supplementary Files}

This is a list of supplementary files associated with this preprint. Click to download.

- SupplementalFig.1.tif 